OPEN ACCESS

Edited by:

Xiongwei Zhu,

Case Western Reserve University,

USA

Reviewed by:

Ying $X u$,

The State University of New York

at Buffalo, USA

Bo Su,

Shandong University, China

${ }^{*}$ Correspondence:

Sheng-Di Chen

chen_sd@medmail.com.cn

tThese authors have contributed equally to this work.

Received: 31 December 2015

Accepted: 22 February 2016

Published: 07 March 2016

Citation:

Huang P, Fang R, Li B-Y and Chen

S-D (2016) Exercise-Related Changes

of Networks in Aging and Mild

Cognitive Impairment Brain.

Front. Aging Neurosci. 8:47.

doi: 10.3389/fnagi.2016.00047

\section{Exercise-Related Changes of Networks in Aging and Mild Cognitive Impairment Brain}

\author{
Pei Huang ${ }^{1 t}$, Rong Fang ${ }^{2 t}$, Bin-Yin Li' and Sheng-Di Chen ${ }^{1 *}$ \\ 1 Department of Neurology and Institute of Neurology, Ruijin Hospital, Shanghai Jiao Tong University School of Medicine, \\ Shanghai, China, ${ }^{2}$ Department of Neurology, Ruijin Hospital and Ruijin Hospital North, Shanghai Jiao Tong University School \\ of Medicine, Shanghai, China
}

Aging and mild cognitive impairment (MCl) are accompanied by decline of cognitive functions. Meanwhile, the most common form of dementia is Alzheimer's disease (AD), which is characterized by loss of memory and other intellectual abilities serious to make difficulties for patients in their daily life. $\mathrm{MCl}$ is a transition period between normal aging and dementia, which has been used for early detection of emerging dementia. It converts to dementia with an annual rate of $5-15 \%$ as compared to normal aging with $1 \%$ rate. Small decreases in the conversion rate of $\mathrm{MCl}$ to $\mathrm{AD}$ might significantly reduce the prevalence of dementia. Thus, it is important to intervene at the preclinical stage. Since there are still no effective drugs to treat $A D$, non-drug intervention is crucial for the prevention and treatment of cognitive decline in aging and $\mathrm{MCl}$ populations. Previous studies have found some cognitive brain networks disrupted in aging and $\mathrm{MCl}$ population, and physical exercise (PE) could effectively remediate the function of these brain networks. Understanding the exercise-related mechanisms is crucial to design efficient and effective PE programs for treatment/intervention of cognitive decline. In this review, we provide an overview of the neuroimaging studies on physical training in normal aging and $\mathrm{MCl}$ to identify the potential mechanisms underlying current physical training procedures. Studies of functional magnetic resonance imaging, electroencephalography, magnetoencephalography and positron emission tomography on brain networks were all included. Based on our review, the default mode network, fronto-parietal network and fronto-executive network are probably the three most valuable targets for efficiency evaluation of interventions.

\footnotetext{
Keywords: exercise, aging, mild cognitive impairment, functional magnetic resonance imaging, electroencephalogram, magnetoencephalography, positron emission tomography
}

\section{INTRODUCTION}

Aging is characterized by a progressive decline of physiological efficiency in cells and tissues that increases the risk of disease and death, which is not pathological but obligatorily normal (Harman, 2001). Decline of cognitive functions, along with structural and functional changes in brain regions, are accompanying symptoms of aging in humans (Hedden and Gabrieli, 2004).

Abbreviations: $A D$, Alzheimer's disease; $A \beta$, amyloid- $\beta$; CSF, cerebrospinal fluid; EEG, electroencephalogram; dACC, dorsal anterior cingulate cortex; DLPFC, dorsolateral prefrontal cortex; DMN, default mode network; ERPs, event-related potentials; fMRI, functional magnetic resonance imaging; FEN, fronto-executive network; FPN, fronto-parietal network; MCI, mild cognitive impairment; MEG, magnetoencephalography; p-tau, phosphorylated tau; PE, physical exercise; $t$-tau, total tau; PET, positron emission tomography. 
Age-related cognitive decline is mainly associated with working memory, executive function and episodic memory (Schaie, 1994). Meanwhile, the most common form of dementia is $\mathrm{AD}$, which is characterized by loss of memory and other intellectual abilities serious to make difficulties for patients in their daily life. AD is not only a cognitive problem, but also a main cause of mortalities in older adults. Until 2013, the number of AD patients has reached 35 million worldwide, and this number is supposed to triple by 2050 (Hosseini et al., 2014; James et al., 2014). However, there are still no effective disease-modifying drugs for AD.

Mild cognitive impairment represents a transitional condition between normal aging and mild dementia, which has been used for early detection of emerging dementia (Petersen et al., 2001, 2009; Guillozet et al., 2003). Indeed, MCI patients already show significant lesion loads (Jack et al., 2013; Villemagne et al., 2013). The most common form of MCI is amnestic mild cognitive impairment (aMCI), which is characterized by slight memory impairment with other cognitive domains being relatively preserved (Petersen et al., 2009; Dubois et al., 2010). It converts to dementia with an annual rate of $5-15 \%$ as compared to normal aging with $1 \%$ rate (Petersen et al., 2009). Previous studies demonstrated that small decreases in the conversion rate of MCI to dementia might significantly reduce the prevalence of dementia (Ferri et al., 2005). Thus, MCI has been recognized as a target for interventions to slow down the progression of cognitive decline to dementia (Petersen and Morris, 2005).

Since the number of dementia is rapidly growing and there are still no effective drugs to treat it, it is necessary to intervene at the preclinical stage. Non-drug intervention is important for the prevention and treatment of cognitive decline of aging and MCI. Many studies suggest that PE, specifically incorporating aerobic exercise, might lead to cognitive improvement in normal aging, MCI and AD (Kramer and Erickson, 2007; Hillman et al., 2008; Lautenschlager et al., 2008; van Uffelen et al., 2008; Baker et al., 2010; Suzuki et al., 2012; Bherer et al., 2013). Exercise can contribute to enhanced blood flow and changes in the brain environment, leading to the restoration of physiological and structural function (Petzinger et al., 2013). Functional MRI data showed that neuronal activity in the prefrontal regions of normal aging and AD became more efficient after aerobic exercise (Colcombe et al., 2004; Angevaren et al., 2008). Aerobic exercise might restore cognition of normal aging and $\mathrm{AD}$ through the promotion of neuroplasticity (Turner and Spreng, 2012). Increasing evidences demonstrated exerciserelated improvements in cognitive performance and revealed exercise-related changes in neuroimaging biomarkers in normal aging, MCI and early stages of AD (Kramer and Erickson, 2007; Hillman et al., 2008; Lautenschlager et al., 2008; van Uffelen et al., 2008; Baker et al., 2010; Suzuki et al., 2012; Bherer et al., 2013). It is important to investigate neural networks that are affected in normal aging and MCI, and those rehabilitated by the training procedure. Understanding the exercise-related mechanisms is crucial to design efficient and effective PE programs for treatment/intervention of cognitive decline.

In this review article, we provide an overview of the neuroimaging studies on physical training in normal aging and MCI to identify the potential mechanisms underlying current physical training procedures. We will then discuss the common findings of the current studies and address the implications for future research.

\section{AGING AND MILD COGNITIVE IMPAIRMENT-RELATED BIOCHEMICAL CHANGES}

The major pathological features in $\mathrm{AD}$ brains are the presence of intra-neurofibrillary tangles and extracellular protein $\mathrm{A} \beta$ deposits, especially in the regions related to learning, memory, and cognitive functions (Reddy and McWeeney, 2006). The distribution of amyloid deposits in MCI was reported to be intermediate between the changes in normal aging and $\mathrm{AD}$ brain (Markesbery, 2010). Growing evidences suggested that aging was a key factor for the increase in production of $A \beta$ and the decreased $\mathrm{A} \beta$-degrading enzymes in the $\mathrm{AD}$ brain (Reddy et al., 2010). Studies of postmortem brains from aged humans with $\mathrm{MCI} / \mathrm{AD}$ found that $\mathrm{A} \beta$ levels increased with age (Gouras et al., 2005). In addition, AD patients differed from normal controls in CSF proteins, with early increase of $A \beta 42$ and later decrease of t-tau and p-tau (Antonell et al., 2011). Those CSF biomarkers also showed good diagnostic accuracy and prediction of conversion from MCI to AD (van Harten et al., 2013; Dubois et al., 2014).

\section{AGING AND MILD COGNITIVE IMPAIRMENT-RELATED CHANGES OF BRAIN NETWORKS}

The field of systematic neuroscience has been revolutionized by functional neuroimaging in recent years. Techniques such as fMRI, EEG, MEG, and PET now provide researchers with unprecedented information on the mechanisms of human brain function. In the following, we provide an overview of neuroimaging studies, which observed brain network changes in normal aging and MCI, to understand the underlying mechanisms of cognitive decline (Table 1).

\section{Functional Magnetic Resonance Imaging}

Previous studies have shown that episodic memory, processing speed, executive function, and other cognitions declined with aging (Hedden and Gabrieli, 2004; Nyberg et al., 2012). FMRI including resting-state or task-based serials is widely used to investigate the relationships between brain networks and cognition. Tambini et al. (2010) performed resting-state fMRI scans in healthy persons, and found enhanced functional connectivity between hippocampus and the lateral occipital complex associated with higher subsequent memory scores after associative encoding tasks. Sala-Llonch et al. (2015) proved that the strength of connectivity between hippocampus and other cortex was weaker with aging (Madden et al., 2010). At the meantime, some studies found that elderly persons had higher activities in some brain domains using task-based fMRI, which reflected a compensatory mechanism when the functional 


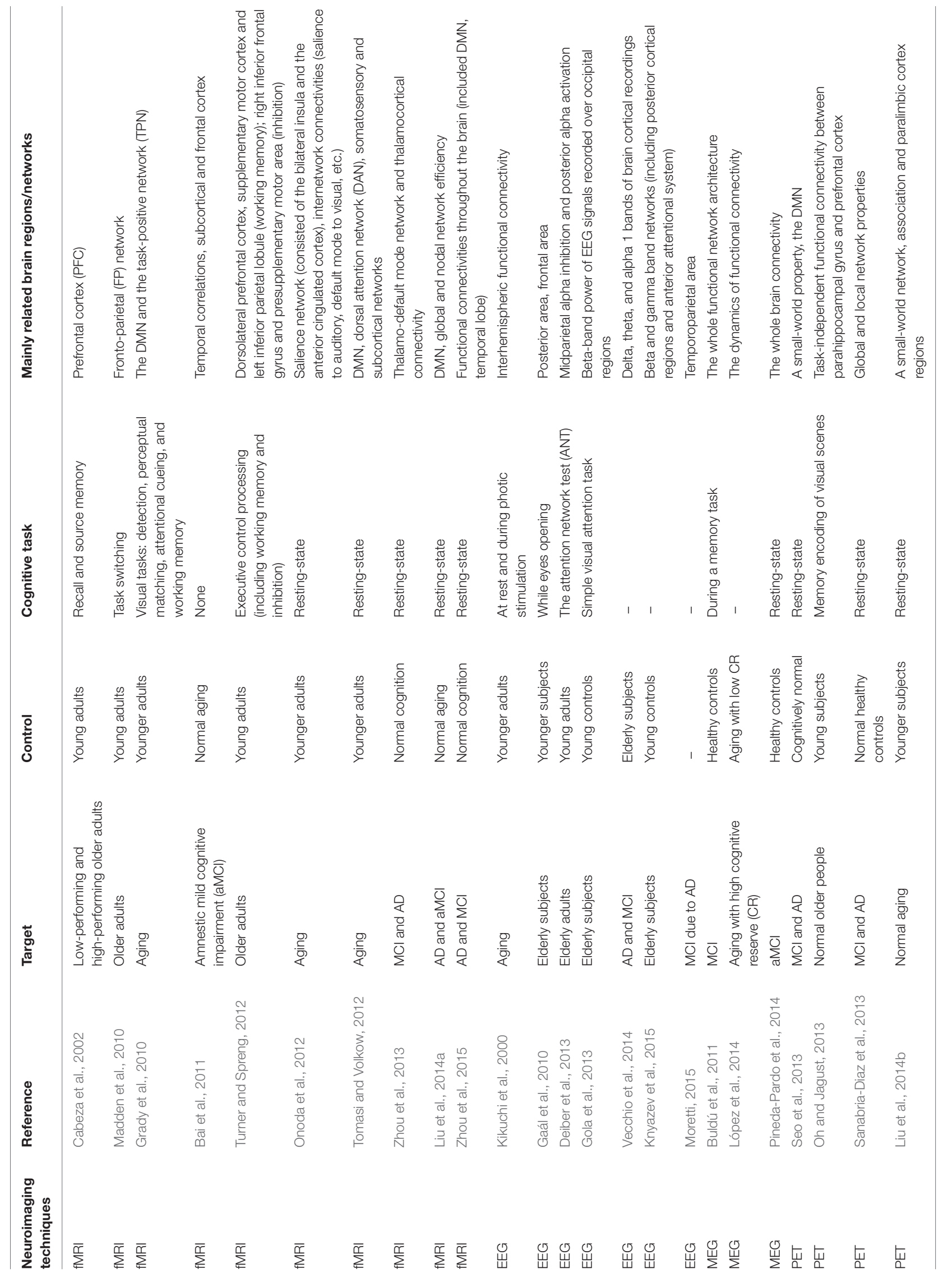


connectivity of brain was disrupted (Grady, 2012). The findings above might explain the mechanisms of poor cognitive functions in aging. But the changes of brain activities in normal aging were very complex. Grady (2012) found that increased brain activity in elderly adults could be associated with either better or worse task performance. The truth is that aging could be influenced by a large number of factors, including education, life experiences, diet, and genes. Nevertheless, many studies have reached an agreement that normal aging population showed altered patterns of brain activity compared to young adults when performing cognitive tasks (Turner and Spreng, 2012). The phenomenon of different patterns of brain activity in aging population could be explained as follows: (1) the differentiation hypothesis: it has been suggested that a decline in dopaminergic neuromodulator could lead to less neural noise, which directly showed less distinctive cortical activities associated with deficits in cognition (Li et al., 2001); (2) the compensation hypothesis: the elderly adults recruited more strength of network than younger adults but inefficiently, especially in prefrontal cortex (Cabeza et al., 2002). In general, task-based fMRI reflects the activities of different brain regions while resting-state fMRI calculates the connectivity magnitude between brain regions. Different models of fMRI can investigate different aspects of the brain networks and we should use them combined.

Mild cognitive impairment is considered as a transition period between normal aging and dementia, especially, the aMCI has a high risk of progression to AD (Petersen et al., 2009). So studies focusing on the brain networks of MCI help us understand the pathological cascading of AD. Some fMRI studies have reported that the strength of functional connectivity, which was positively correlated with the scores of the mini-mental state examination (MMSE), was weaker in MCI than normal aging (Bai et al., 2011; Zhou et al., 2013, 2015). Bai et al. (2011) revealed abnormal interregional correlations in the whole brain areas of MCI patients when compared to normal aging populations, particularly in subcortical regions and frontal cortex. In addition, they also found that negative functional connections decreased with the progression of disease (Bai et al., 2011). There may be a compensatory mechanism during the process of MCI as in normal aging. Besides, Liu et al. (2014a) proved that the loss of long-distance connectivity was related to the severity of MCI and AD (Liu et al., 2014a).

The most commonly studied brain network using resting-state fMRI is the DMN, which has the advantage in reflecting internal cognition at rest. DMN is the most relevant network model reflecting the functional and pathological cascade underlying AD (Buckner et al., 2009). Buckner et al. (2008) found that the DMN mainly included the medial temporal lobe and the medial prefrontal subsystems. There was evidence that the DMN was overlapped with brain areas that had the deposition of CSF biomarkers, including $A \beta 42$, t-tau and p-tau (Li et al., 2014). Functional connectivities within the DMN were found reduced in older adults compared with the younger adults (Grady et al., 2010). This helps us further understand MCI and AD, which are both described as disconnective syndromes (Seeley et al., 2009). Besides DMN, other intrinsic brain networks have also been studied in aging. Yeo et al. (2011) organized the cortex into seven networks coarsely and 17 networks at a finer level according to 1,000 individuals. Onoda et al. (2012) reported that salience network and other internetworks were also disrupted in elders, which were related to cognitive decline. Tomasi reported that aging had severer impact on the long-range functional connectivity density (FCD) than on the short-range FCD, indicating that long-range networks might be more vulnerable to aging (Tomasi and Volkow, 2012). Similarly, anterior and posterior components of the DMN, which are long-distance connectivities, are destructed in MCI patients. And severer reduction of global and nodal network efficiency was found in MCI and AD compared to normal aging (Liu et al., 2014a). Consistent with previous results, the connectivities of several important nodes within the DMN in MCI and AD patients had been found inefficient by Zhou et al. (2015) such as the posterior cingulate gyrus, the precuneus, the parahippocampal gyrus, and the medial superior frontal gyrus. These findings support that human brain is the integration of neural networks and MCI is a prodromal stage of AD (Gauthier et al., 2006).

\section{Electroencephalogram}

EEG is a useful tool in detecting cognitive level and brain networks (Gaál et al., 2010). van der Hiele et al. (2008) reported that baseline EEG, which showed increased theta activity (4-8 Hz) during eyes closed and less alpha reactivity $(8-13 \mathrm{~Hz})$ during eyes open and memory activation, could be markers of future cognitive level of the elderly. Kikuchi et al. (2000) found that there was a lower coherence in the resting EEG for the delta, theta, alpha-3, beta- 1 and beta- 2 frequency bands, which reflected a weaker interhemispheric functional connectivity in elderly subjects. Gaál et al. (2010) pointed out decreased values of the clustering coefficient, path length and the "small-world index" when elders were opening eyes compared with younger subjects. It also suggested a reduced reactivity with aging and a decreased level of integrative activity of the brain (Gaál et al., 2010). Besides, an overall reduction of task-related alpha activity in brain circuits was found in the elderly compared with the youngers during attentional functions (Deiber et al., 2013). The beta-band activity of EEG in elderly subjects was also found to be decreased, which proved a deficit of aging population in attention processes (Gola et al., 2013). Furthermore, Knyazev et al. (2015) found a decrease of modularity and clustering in beta and gamma band networks in aging using Graph-theoretical analysis with EEG, which implied that aging made brain networks more randomly.

EEG has also been used to detect the brain cognitive networks of MCI patients. Vecchio et al. (2014) discovered that MCI subjects were significantly impaired in theta but not alpha bands connectivity compared with normal aging with graph theoretical analysis. A study of EEG revealed that upper/low alpha power ratio could predict $\mathrm{MCI}$, which was associated with cortical thinning and less perfusion in the temporoparietal area (Moretti, 2015).

\section{Magnetoencephalography}

MEG is another important tool to study the brain networks and cognitive functions of aging and MCI, which records the neuronal activity reflecting the dynamics of the cortical networks 
(López et al., 2014). A study of the neural activities using MEG indicated that alpha oscillatory activity in DLPFC was synchronized with that in parietal regions during visual shortterm memory (Grimault et al., 2009). Besides, it was verified that parieto-occipital alpha power was markedly stronger for successfully encoded long-term memory sequences (Meeuwissen et al., 2011). A research of aging populations revealed that the subjects with lower cognitive reserve exhibited higher functional connectivity than those with higher cognitive reserve in MEG signals (López et al., 2014). This can be explained by a way of compensation.

In the MEG study of brain networks in MCI, Buldú et al. (2011) reported that MCI patients showed an enhancement of the strength of connections, together with an increase in the outreach parameter. In addition, scientists proposed a structural connectivity of the graphical lasso (GL), in which region-specific time series were obtained in five different frequency bands. The results concluded that the structural connectivity could estimate functional activity, and this classification method of MCI was better than traditional ways (Pineda-Pardo et al., 2014).

\section{Positron Emission Tomography}

Positron emission tomography is also an important tool to investigate the brain networks, which reflects the cerebral glucose metabolism sensitively (Liu et al., 2014b). Previous study certified that the index of blood-flow tested by PET was consistent with brain anatomical connectivity (Ferrarelli et al., 2004). Liu et al. (2014b) reported that both of younger adults and elderly subjects showed small-world architecture in brain networks utilizing PET, but increased clustering and decreased efficiency were found in elderly subjects. Taking advantage of Pittsburg compound B-positron emission tomography (PiB-PET), Oh and Jagust (2013) certified that elderly adults without $\mathrm{A} \beta$ deposition showed decreased regional brain activation and decreased functional connectivity between parahippocampal gyrus and prefrontal cortex compared with young subjects.

PET with ${ }^{18} \mathrm{~F}$-fluorodeoxyglucose ([ $\left.\left.{ }^{18} \mathrm{~F}\right] \mathrm{FDG}-\mathrm{PET}\right)$, which is a marker of synaptic function, measures regional brain glucose metabolism (rBGM). Hypometabolism on $\left[{ }^{18} \mathrm{~F}\right] \mathrm{FDG}-\mathrm{PET}$ is related to neuronal dysfunction and neurodegeneration (Herholz et al., 2011; Jack et al., 2013). A reduction in rBGM in the posterior cingulate and temporoparietal regions was related to a faster cognitive decline in MCI patients (Minoshima et al., 1997; Drzezga et al., 2003). Seo et al. (2013) reported that MCI and $\mathrm{AD}$ had the same 'small-world' property of whole-brain network as cognitively normal populations using $\left[{ }^{18} \mathrm{~F}\right]$ FDG-PET. However, local clustering of networks was lower and normalized betweenness centrality in hubs of DMN was decreasing in MCI compared to cognitively normal populations (Seo et al., 2013). Besides, Sanabria-Diaz et al. (2013) detected the global and local network properties (global and local efficiency, clustering index, and others) in MCI using $\left[{ }^{18} \mathrm{~F}\right]$ FDG-PET, and certified that the network of MCI was in the transitional stage between cognitively normal populations and $\mathrm{AD}$.

\section{EXERCISE-RELATED CHANGES OF NETWORKS IN AGING AND MILD COGNITIVE IMPAIRMENT}

Exercise has been found to lead to cognitive improvement in normal aging, MCI and $\mathrm{AD}$ with increasing evidences demonstrated exercise-related changes in imaging biomarkers. In order to understand the exercise-related mechanisms underlying cognitive improvements, we provide an overview of neuroimaging studies focusing on brain network changes caused by exercise in normal aging and MCI (Table 2).

\section{Functional Magnetic Resonance Imaging}

Functional MRI data have demonstrated exercise-related brain network changes in cognitive regions of healthy persons. Higher connectivity within the DMN has been associated with increased cardiorespiratory fitness, and DMN connectivity also mediates the relationship between maximal oxygen consumption $\left(\mathrm{VO}_{2}\right.$ max) and cognitive functions (Voss et al., 2010a). Another study, which examined the effect of aerobic fitness on cognitive control in preadolescent children, found that highfit children outperformed low-fit children on cognitive control, and individual differences in cognitive control performance were associated with aerobic fitness (Voss et al., 2011). Longitudinal functional and structural MRI study of young adults who accepted a 6-week motor training showed increased FPN connectivity in accordance with cognitive performance improvements. The structural gray matter alterations were also tightly correlated with functional connectivity changes in prefrontal and supplementary-motor areas (Taubert et al., 2011).

Studies on older adults also found that age-related dysfunction of brain networks were remediated by PE. Zlatar et al. (2013) found that during a semantic fluency task, inactive aging displayed reductions in negative task-related activity compared to the active aging in areas of the attention network. It indicated that exercise might remediate these alterations in network activity related to attention and language processing, physical activity may alleviate the impact of aging on language functions (Zlatar et al., 2013). Wei et al. (2014) found that Tai Chi Chuan (TCC) could influence the functional plasticity of the brain's intrinsic architecture and optimize locally functional organization to improve cognition in aging population. Moreover, TCC also potentially increased the thickness of brain regions associated with motor and executive functions (Wei et al., 2013). After a 1-year exercise intervention in aging adults, both the DMN and the FPN exhibited higher connectivity than controls (Voss et al., 2010b).

Furthermore, some studies revealed changes in imaging biomarkers in subjects with MCI who performed PE. One study used structural MRI to reveal a lower rate of brain atrophy in the MCI population (Suzuki et al., 2013). Another study used functional MRI to show decreases in hippocampal activation during a semantic memory retrieval task, indicating that neural efficiency was improved after the PE intervention 

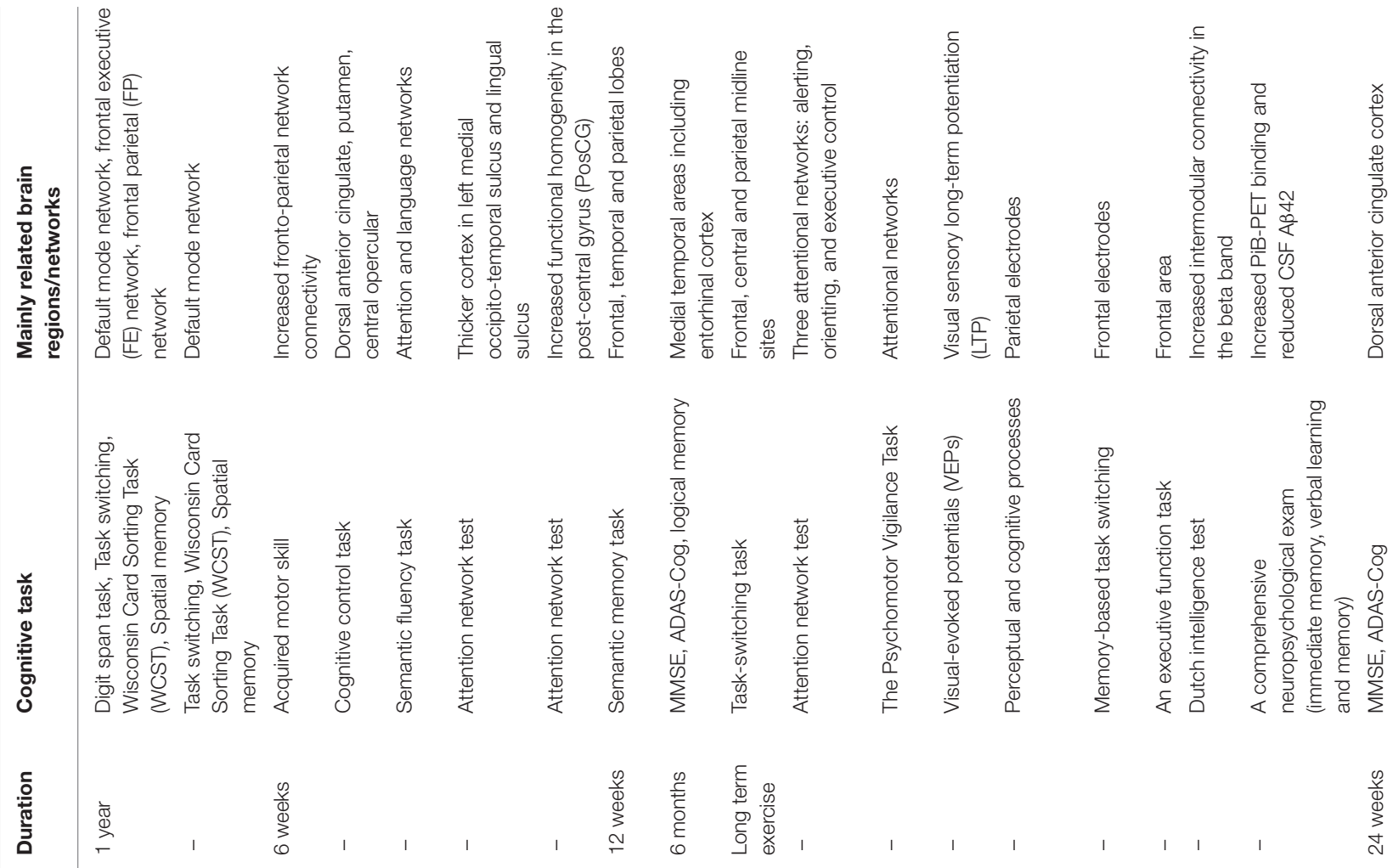

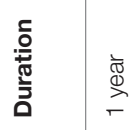
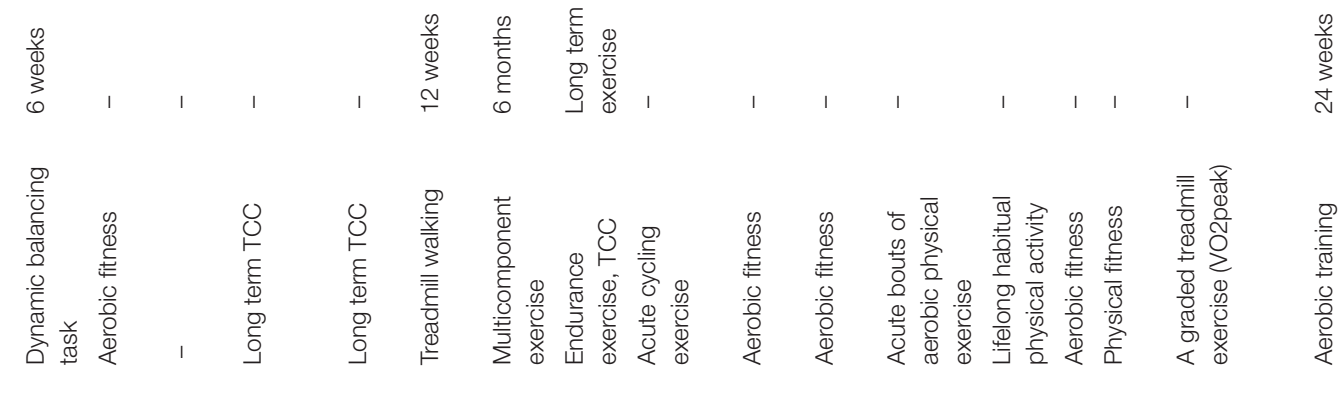

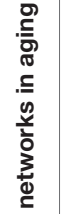

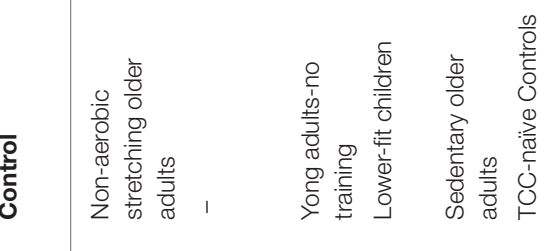

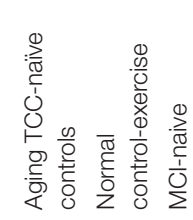
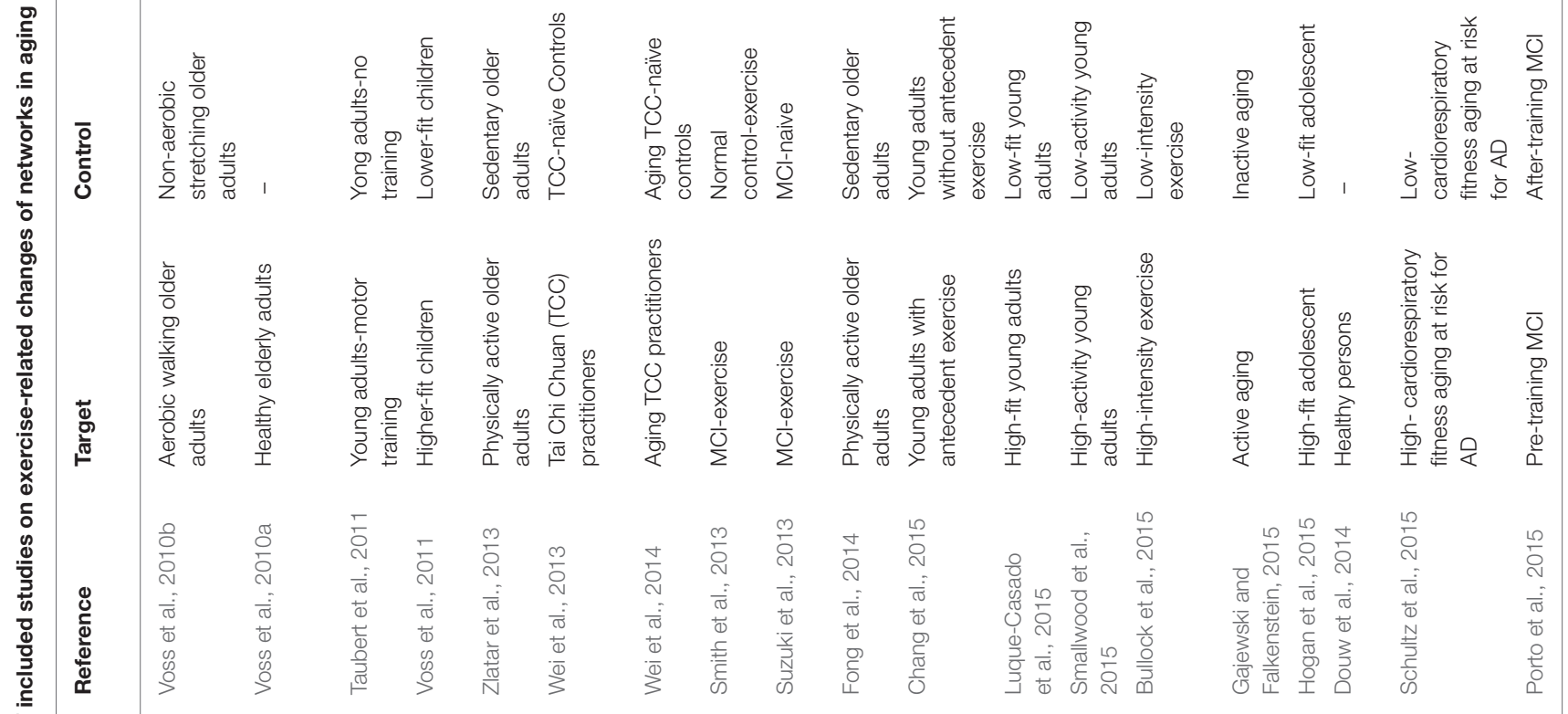

int
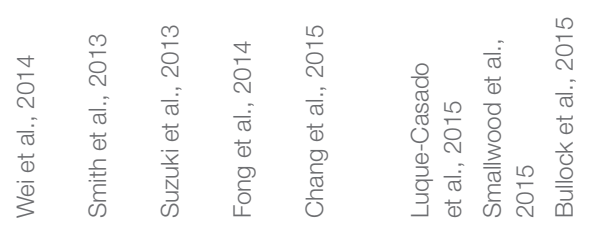

影影

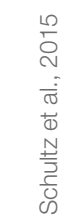


(Smith et al., 2013). Overall, exercises bring improvements in cognitive function accompanied by functional and structural changes in brain regions both in normal aging and MCI.

\section{Electroencephalogram}

EEG and ERPs provide higher temporal-resolution biomarkers than neuroimages for cognitive changes. Hogan et al. (2015) measured EEG entropy to uncover the effect of physical fitness on executive function. It was suggested that the effect came from higher functionality of the attentional system in the context of lower levels of frontal EEG entropy. The study repeatedly measured changes in entropy during the $1500 \mathrm{~ms}$ post-stimulus interval. ERPs provide closely relationship between cognitive process and electroneurophysiological changes. Regarding the attentional system, Fong et al. (2014) observed P300 amplitude between young adults and older adults with endurance exercises, TCC or sedentary life-style. Compared with older adults with sedentary lifestyle, all other three groups had significantly larger P3 amplitude in task-switching task (Fong et al., 2014). The author concluded that age and participation in physical activity influenced the relationship between physical activity and taskswitching (Fong et al., 2014). Attentional network improvement was also shown in aerobic cycling exercise. It resulted in a larger P3 amplitude in the alerting and executive control subtasks across frontal, central and parietal midline sites (Chang et al., 2015). In a psychomotor vigilance task, behavioral and electrophysiological ERPs were obtained and analyzed as a function of time-ontask. Higher-fit participants maintained larger P3 amplitude throughout the task compared to lower-fit who showed a reduction in the P3 magnitude over time (Luque-Casado et al., 2015). Learning is a crucial process in cognitive ability. Long-term potentiation (LTP) represented network plasticity, and worked as an enhancer for learning. In one study, high-activity group maintained amplitude of the $\mathrm{N} 1 \mathrm{~b}$ after a 30 -min rest period, while low-activity group returned to baseline (Smallwood et al., 2015).

Not only one cognitive domain was affected by PE. The amplitude and latency of the visual P1 component and P3a ERPs component evoked in the Oddyball paradigm differed in low and high-intensity exercise group. It was suggested that exercise modulated multiple stages of neural information processing, ranging from early stage sensory processing (P1) to post-perceptual target categorization (P3a) (Bullock et al., 2015). In a retrospective study for aging and physical activity, 50years lifelong physical activity was associated with faster recall of stimulus-response sets (P2), enhanced response selection during interference processing (N2) and working memory updating (P3b) leading to lower mixing and switch costs (Gajewski and Falkenstein, 2015).

However, cognitive impairment was also observed during both low and moderate-intensity exercise for the flanker task trials that require greater cognitive control. Interestingly, ERPs revealed increased $\mathrm{N} 2$ and $\mathrm{P} 3$ amplitudes during both exercise conditions relative to rest. The author suggested divergent effects of exercise on behavioral performance measures, accompanied by an upregulation of cognitive control during aerobic exercises (Olson et al., 2015). The study only evaluated the effect of 3-day exercise in healthy participants, regardless of long-term effect.

\section{Magnetoencephalography}

Functional connectivity can also be determined from MEG, which is a much more direct way to measure neural activity. The brain network is a 'smallworld,' which combined local segregation with global integration (Watts and Strogatz, 1998; Sporns and Zwi, 2004; Stam, 2004; Bassett et al., 2006). Brain network topology is disturbed in aging and MCI (Bullmore and Sporns, 2009; Stam and van Straaten, 2012). Increased physical fitness was related to better functional brain network topology. Douw et al. (2014) proved that physical fitness was related to modular network topology based on MEG in healthy subjects. The increased intermodular connectivity was associated with better cardio respiratory fitness and better mental fitness, while having less within-module connections. Thus, MEG also showed exercise-related improvements in brain network functions.

\section{Positron Emission Tomography}

Hypometabolism in the DMN is an important $\left[{ }^{18} \mathrm{~F}\right]$ FDG-PET marker for the progression of MCI to dementia and has been considered the "metabolic property" of AD (Minoshima et al., 1997; Drzezga et al., 2003; Jack et al., 2013; Fjell et al., 2014). In a study of MCI persons, authors evaluated the effects of a 24week PE on cognition and rBGM using $\left[{ }^{18} \mathrm{~F}\right] \mathrm{FDG}-\mathrm{PET}$. Brain metabolic analysis found a bilateral decrease in the rBGM of the dACC, which was negatively correlated with improvements in a visuospatial function/attentional task (Porto et al., 2015). In addition, a study of aging at risk of AD by PiB-PET imaging found that higher cardiorespiratory fitness was related to better cognition, with increased PiB-PET binding and reduced $\mathrm{A} \beta$ in CSF, indicating lower risk of developing into dementia (Schultz et al., 2015). Overall, PE improves cognition and changes metabolic networks in areas related to cognition in subjects at risk of dementia.

\section{POTENTIAL TARGETS FOR INTERVENTIONS OF COGNITIVE DECLINE}

From our view of imaging studies on aging and MCI-related changes in brain networks, and exercise-reduced alterations in brain networks, we identify three important networks that might be potential targets for intervention of cognitive decline. The three networks are DMN, FPN and FEN.

\section{Default Mode Network}

The DMN is composed of the posterior cingulate, ventral and superior frontal medial cortices, and bilateral lateral occipital, middle frontal, hippocampal and parahippocampal, and middle temporal cortices (Fox et al., 2005; Buckner et al., 2008). The DMN is supposed to have an important functional role in memory consolidation, self-referential thought, mindwandering, autobiographical memory (Buckner et al., 2008; Schilbach et al., 2008), and executive control. Increased DMN function has been related to better working memory in young adults (Hampson et al., 2006), and better executive function in 
older adults (Andrews-Hanna et al., 2007; Persson et al., 2007; Damoiseaux et al., 2008; Voss et al., 2010a), indicating that $\mathrm{DMN}$ is an important network for understanding age-related changes in cognition. In addition, DMN is the most relevant network model reflecting the functional and pathological cascade underlying AD (Buckner et al., 2009). The connectivities of the DMN in MCI and AD have been found inefficient (Zhou et al., 2015). However, previous studies showed that exercise could enhance the connectivity of the DMN. Higher connectivity within the DMN had been related to increased cardiorespiratory fitness and exercise intervention increased the DMN connectivity (Voss et al., 2010a,b). Thus, DMN network is a crucial target for interventions of aging and MCI. In order to evaluate the effectiveness and efficiency of PE program, we must pay more attention to the function of the DMN.

\section{Fronto-Parietal Network and Fronto-Executive Network}

Special attention should also be paid to the FPN and the FEN. The inferior parietal cortices, the supplementary motor and primary cortices, the frontal eye-fields, primary and extrastriate visual cortices, the inferior frontal cortex are included in the FPN (Corbetta and Shulman, 2002; Dosenbach et al., 2006). Age-related structural and functional disruptions of the FPN have been found in some studies (Andrews-Hanna et al., 2007; Madden et al., 2007) and could be remediated by PE. The FPN has some overlapping portions with the FEN at the tempoparietal junction. The FEN is associated with sustained task-set maintenance, error feedback for tuning top-down control, and maintaining action-outcome associations (Rushworth et al., 2004; Dosenbach et al., 2006). It is composed of the anterior prefrontal cortex, insular and frontal operculum cortices, the tempoparietal junction, and the dorsal posterior and anterior cingulate gyri (Dosenbach et al., 2006). Age-related cognitive decline in learning tasks have been found related to the dysfunction of this network

\section{REFERENCES}

Andrews-Hanna, J. R., Snyder, A. Z., Vincent, J. L., Lustig, C., Head, D., Raichle, M. E., et al. (2007). Disruption of large-scale brain systems in advanced aging. Neuron 56, 924-935. doi: 10.1016/j.neuron.2007.10.038

Angevaren, M., Aufdemkampe, G., Verhaar, H. J., Aleman, A., and Vanhees, L. (2008). Physical activity and enhanced fitness to improve cognitive function in older people without known cognitive impairment. Cochrane Database Syst. Rev. 2, CD005381. doi: 10.1002/14651858.CD0 05381.pub3

Antonell, A., Fortea, J., Rami, L., Bosch, B., Balasa, M., Sanchez-Valle, R., et al. (2011). Different profiles of Alzheimer's disease cerebrospinal fluid biomarkers in controls and subjects with subjective memory complaints. J. Neural Transm. (Vienna) 118, 259-262. doi: 10.1007/s00702-010-0534-0

Bai, F., Liao, W., Watson, D. R., Shi, Y., Wang, Y., Yue, C., et al. (2011). Abnormal whole-brain functional connection in amnestic mild cognitive impairment patients. Behav. Brain Res. 216, 666-672. doi: 10.1016/j.bbr.2010.09.010

Baker, L. D., Frank, L. L., Foster-Schubert, K., Green, P. S., Wilkinson, C. W., McTiernan, A., et al. (2010). Effects of aerobic exercise on mild cognitive impairment: a controlled trial. Arch. Neurol. 67, 71-79. doi: 10.1001/archneurol.2009.307

Bassett, D. S., Meyer-Lindenberg, A., Achard, S., Duke, T., and Bullmore, E. (2006). Adaptive reconfiguration of fractal small-world human brain
(Park and Reuter-Lorenz, 2009), while exercise could alleviate cognitive performance and brain network functionality. Thus, FPN and FEN are important index for the efficiency evaluation of interventions of cognitive decline.

\section{FUTURE DIRECTIONS}

In the future, longitudinal brain imaging data and the combination of powerful network computational algorithms may generate a new class of progression biomarkers for preclinical dementia. In addition to generating important translational data regarding the systems-level changes that underlie preclinical disease progression, exercise-related changes in functional brain networks may prove efficient and effective PE programs for interventions of cognitive decline. Based on our review, the DMN, FPN and FEN are probably the three most valuable targets for efficiency evaluation of cognitive decline interventions.

\section{AUTHOR CONTRIBUTIONS}

S-DC designed the whole study and gave suggestions on revising the article. PH and RF searched and selected the studies, analyzed the data, drafted and revised the article. B-YL did some part of writing in EEG data and revised the article. All authors read and approved the final manuscript.

\section{FUNDING}

This work was supported by grants from the National Natural Science Foundation of China (91332107, 30872729, 30971031, 81400888) and The Training and Financial Program for Young Doctors of Shanghai (20141060).

functional networks. Proc. Natl. Acad. Sci. U.S.A. 103, 19518-19523. doi: 10.1073/pnas.0606005103

Bherer, L., Erickson, K. I., and Liu-Ambrose, T. (2013). A review of the effects of physical activity and exercise on cognitive and brain functions in older adults. J. Aging Res. 2013, 657508. doi: 10.1155/2013/ 657508

Buckner, R. L., Andrews-Hanna, J. R., and Schacter, D. L. (2008). The brain's default network: anatomy, function, and relevance to disease. Ann. N. Y. Acad. Sci. 1124, 1-38. doi: 10.1196/annals.1440.011

Buckner, R. L., Sepulcre, J., Talukdar, T., Krienen, F. M., Liu, H., Hedden, T., et al. (2009). Cortical hubs revealed by intrinsic functional connectivity: mapping, assessment of stability, and relation to Alzheimer's disease. J. Neurosci. 29, 1860-1873. doi: 10.1523/jneurosci.5062-08.2009

Buldú, J. M., Bajo, R., Maestu, F., Castellanos, N., Leyva, I., Gil, P., et al. (2011). Reorganization of functional networks in mild cognitive impairment. PLoS ONE 6:e19584. doi: 10.1371/journal.pone.0019584

Bullmore, E., and Sporns, O. (2009). Complex brain networks: graph theoretical analysis of structural and functional systems. Nat. Rev. Neurosci. 10, 186-198. doi: $10.1038 / \mathrm{nrn} 2575$

Bullock, T., Cecotti, H., and Giesbrecht, B. (2015). Multiple stages of information processing are modulated during acute bouts of exercise. Neuroscience 307, 138-150. doi: 10.1016/j.neuroscience.2015. 08.046 
Cabeza, R., Anderson, N. D., Locantore, J. K., and McIntosh, A. R. (2002). Aging gracefully: compensatory brain activity in high-performing older adults. Neuroimage 17, 1394-1402. doi: 10.1006/nimg.2002.1280

Chang, Y. K., Pesce, C., Chiang, Y. T., Kuo, C. Y., and Fong, D. Y. (2015). Antecedent acute cycling exercise affects attention control: an ERP study using attention network test. Front. Hum. Neurosci. 9:156. doi: 10.3389/fnhum.2015.00156

Colcombe, S. J., Kramer, A. F., McAuley, E., Erickson, K. I., and Scalf, P. (2004). Neurocognitive aging and cardiovascular fitness: recent findings and future directions. J. Mol. Neurosci. 24, 9-14. doi: 10.1385/JMN:24:1:009

Corbetta, M., and Shulman, G. L. (2002). Control of goal-directed and stimulus-driven attention in the brain. Nat. Rev. Neurosci. 3, 201-215. doi: $10.1038 /$ nrn755

Damoiseaux, J. S., Beckmann, C. F., Arigita, E. J., Barkhof, F., Scheltens, P., Stam, C. J., et al. (2008). Reduced resting-state brain activity in the "default network" in normal aging. Cereb. Cortex 18, 1856-1864. doi: 10.1093/cercor/bhm207

Deiber, M. P., Ibanez, V., Missonnier, P., Rodriguez, C., and Giannakopoulos, P. (2013). Age-associated modulations of cerebral oscillatory patterns related to attention control. Neuroimage 82, 531-546. doi: 10.1016/j.neuroimage.2013.06.037

Dosenbach, N. U., Visscher, K. M., Palmer, E. D., Miezin, F. M., Wenger, K. K., Kang, H. C., et al. (2006). A core system for the implementation of task sets. Neuron 50, 799-812. doi: 10.1016/j.neuron.2006.04.031

Douw, L., Nieboer, D., van Dijk, B. W., Stam, C. J., and Twisk, J. W. (2014). A healthy brain in a healthy body: brain network correlates of physical and mental fitness. PLoS ONE 9:e88202. doi: 10.1371/journal.pone.0088202

Drzezga, A., Lautenschlager, N., Siebner, H., Riemenschneider, M., Willoch, F., Minoshima, S., et al. (2003). Cerebral metabolic changes accompanying conversion of mild cognitive impairment into Alzheimer's disease: a PET follow-up study. Eur. J. Nucl. Med. Mol. Imaging 30, 1104-1113. doi: 10.1007/s00259-003-1194-1

Dubois, B., Feldman, H. H., Jacova, C., Cummings, J. L., Dekosky, S. T., BarbergerGateau, P., et al. (2010). Revising the definition of Alzheimer's disease: a new lexicon. Lancet Neurol. 9, 1118-1127. doi: 10.1016/s1474-4422(10)70223-4

Dubois, B., Feldman, H. H., Jacova, C., Hampel, H., Molinuevo, J. L., Blennow, K., et al. (2014). Advancing research diagnostic criteria for Alzheimer's disease: the IWG-2 criteria. Lancet Neurol. 13, 614-629. doi: 10.1016/s1474-4422(14) 70090-0

Ferrarelli, F., Haraldsson, H. M., Barnhart, T. E., Roberts, A. D., Oakes, T. R., Massimini, M., et al. (2004). A [17F]-fluoromethane PET/TMS study of effective connectivity. Brain Res. Bull. 64, 103-113. doi: 10.1016/j.brainresbull.2004.04.020

Ferri, C. P., Prince, M., Brayne, C., Brodaty, H., Fratiglioni, L., Ganguli, M., et al. (2005). Global prevalence of dementia: a Delphi consensus study. Lancet 366, 2112-2117. doi: 10.1016/s0140-6736(05)67889-0

Fjell, A. M., McEvoy, L., Holland, D., Dale, A. M., and Walhovd, K. B. (2014). What is normal in normal aging? Effects of aging, amyloid and Alzheimer's disease on the cerebral cortex and the hippocampus. Prog. Neurobiol. 117, 20-40. doi: 10.1016/j.pneurobio.2014.02.004

Fong, D. Y., Chi, L. K., Li, F., and Chang, Y. K. (2014). The benefits of endurance exercise and Tai Chi Chuan for the task-switching aspect of executive function in older adults: an ERP study. Front. Aging Neurosci. 6:295. doi: 10.3389/fnagi.2014.00295

Fox, M. D., Snyder, A. Z., Vincent, J. L., Corbetta, M., Van Essen, D. C., and Raichle, M. E. (2005). The human brain is intrinsically organized into dynamic, anticorrelated functional networks. Proc. Natl. Acad. Sci. U.S.A. 102, 9673-9678. doi: 10.1073/pnas.0504136102

Gaál, Z. A., Boha, R., Stam, C. J., and Molnar, M. (2010). Age-dependent features of EEG-reactivity-spectral, complexity, and network characteristics. Neurosci. Lett. 479, 79-84. doi: 10.1016/j.neulet.2010.05.037

Gajewski, P. D., and Falkenstein, M. (2015). Lifelong physical activity and executive functions in older age assessed by memory based task switching. Neuropsychologia 73, 195-207. doi: 10.1016/j.neuropsychologia.2015.04.031

Gauthier, S., Reisberg, B., Zaudig, M., Petersen, R. C., Ritchie, K., Broich, K., et al. (2006). Mild cognitive impairment. Lancet 367, 1262-1270. doi: 10.1016/s01406736(06)68542-5

Gola, M., Magnuski, M., Szumska, I., and Wrobel, A. (2013). EEG beta band activity is related to attention and attentional deficits in the visual performance of elderly subjects. Int. J. Psychophysiol. 89, 334-341. doi: 10.1016/j.ijpsycho.2013.05.007

Gouras, G. K., Almeida, C. G., and Takahashi, R. H. (2005). Intraneuronal Abeta accumulation and origin of plaques in Alzheimer's disease. Neurobiol. Aging 26, 1235-1244. doi: 10.1016/j.neurobiolaging.2005.05.022

Grady, C. (2012). The cognitive neuroscience of ageing. Nat. Rev. Neurosci. 13, 491-505. doi: 10.1038/nrn3256

Grady, C. L., Protzner, A. B., Kovacevic, N., Strother, S. C., Afshin-Pour, B., Wojtowicz, M., et al. (2010). A multivariate analysis of age-related differences in default mode and task-positive networks across multiple cognitive domains. Cereb. Cortex 20, 1432-1447. doi: 10.1093/cercor/bhp207

Grimault, S., Robitaille, N., Grova, C., Lina, J. M., Dubarry, A. S., and Jolicoeur, P. (2009). Oscillatory activity in parietal and dorsolateral prefrontal cortex during retention in visual short-term memory: additive effects of spatial attention and memory load. Hum. Brain Mapp. 30, 3378-3392. doi: 10.1002/ hbm.20759

Guillozet, A. L., Weintraub, S., Mash, D. C., and Mesulam, M. M. (2003). Neurofibrillary tangles, amyloid, and memory in aging and mild cognitive impairment. Arch. Neurol. 60, 729-736. doi: 10.1001/archneur.60.5.729

Hampson, M., Driesen, N. R., Skudlarski, P., Gore, J. C., and Constable, R. T. (2006). Brain connectivity related to working memory performance. J. Neurosci. 26, 13338-13343. doi: 10.1523/jneurosci.3408-06.2006

Harman, D. (2001). Aging: overview. Ann. N. Y. Acad. Sci. 928, 1-21. doi: 10.1111/j.1749-6632.2001.tb05631.x

Hedden, T., and Gabrieli, J. D. (2004). Insights into the ageing mind: a view from cognitive neuroscience. Nat. Rev. Neurosci. 5, 87-96. doi: 10.1038/nrn1323

Herholz, K., Westwood, S., Haense, C., and Dunn, G. (2011). Evaluation of a calibrated (18)F-FDG PET score as a biomarker for progression in Alzheimer disease and mild cognitive impairment. J. Nucl. Med. 52, 1218-1226. doi: 10.2967/jnumed.111.090902

Hillman, C. H., Erickson, K. I., and Kramer, A. F. (2008). Be smart, exercise your heart: exercise effects on brain and cognition. Nat. Rev. Neurosci. 9, 58-65. doi: $10.1038 / \mathrm{nrn} 2298$

Hogan, M. J., O'Hora, D., Kiefer, M., Kubesch, S., Kilmartin, L., Collins, P., et al. (2015). The effects of cardiorespiratory fitness and acute aerobic exercise on executive functioning and EEG entropy in adolescents. Front. Hum. Neurosci. 9:538. doi: 10.3389/fnhum.2015.00538

Hosseini, S. M., Kramer, J. H., and Kesler, S. R. (2014). Neural correlates of cognitive intervention in persons at risk of developing Alzheimer's disease. Front. Aging Neurosci. 6:231. doi: 10.3389/fnagi.2014.00231

Jack, C. R. Jr., Knopman, D. S., Jagust, W. J., Petersen, R. C., Weiner, M. W., Aisen, P. S., et al. (2013). Tracking pathophysiological processes in Alzheimer's disease: an updated hypothetical model of dynamic biomarkers. Lancet Neurol. 12, 207-216. doi: 10.1016/s1474-4422(12)70291-0

James, B. D., Leurgans, S. E., Hebert, L. E., Scherr, P. A., Yaffe, K., and Bennett, D. A. (2014). Contribution of Alzheimer disease to mortality in the United States. Neurology 82, 1045-1050. doi: 10.1212/wnl.0000000000000240

Kikuchi, M., Wada, Y., Koshino, Y., Nanbu, Y., and Hashimoto, T. (2000). Effect of normal aging upon interhemispheric EEG coherence: analysis during rest and photic stimulation. Clin. Electroencephalogr. 31, 170-174. doi: $10.1177 / 155005940003100210$

Knyazev, G. G., Volf, N. V., and Belousova, L. V. (2015). Age-related differences in electroencephalogram connectivity and network topology. Neurobiol. Aging 36, 1849-1859. doi: 10.1016/j.neurobiolaging.2015.02.007

Kramer, A. F., and Erickson, K. I. (2007). Capitalizing on cortical plasticity: influence of physical activity on cognition and brain function. Trends Cogn. Sci. 11, 342-348. doi: 10.1016/j.tics.2007.06.009

Lautenschlager, N. T., Cox, K. L., Flicker, L., Foster, J. K., van Bockxmeer, F. M., Xiao, J., et al. (2008). Effect of physical activity on cognitive function in older adults at risk for Alzheimer disease: a randomized trial. JAMA 300, 1027-1037. doi: $10.1001 /$ jama.300.9.1027

Li, S. C., Lindenberger, U., and Sikstrom, S. (2001). Aging cognition: from neuromodulation to representation. Trends Cogn. Sci. 5, 479-486. doi: 10.1016/S1364-6613(00)01769-1

Li, X., Li, T. Q., Andreasen, N., Wiberg, M. K., Westman, E., and Wahlund, L. O. (2014). The association between biomarkers in cerebrospinal fluid and structural changes in the brain in patients with Alzheimer's disease. J. Intern. Med. 275, 418-427. doi: 10.1111/joim.12164 
Liu, Y., Yu, C., Zhang, X., Liu, J., Duan, Y., Alexander-Bloch, A. F., et al. (2014a). Impaired long distance functional connectivity and weighted network architecture in Alzheimer's disease. Cereb. Cortex 24, 1422-1435. doi: $10.1093 /$ cercor/bhs410

Liu, Z., Ke, L., Liu, H., Huang, W., and Hu, Z. (2014b). Changes in topological organization of functional PET brain network with normal aging. PLoS ONE 9:e88690. doi: 10.1371/journal.pone.0088690

López, M. E., Aurtenetxe, S., Pereda, E., Cuesta, P., Castellanos, N. P., Bruna, R., et al. (2014). Cognitive reserve is associated with the functional organization of the brain in healthy aging: a MEG study. Front. Aging Neurosci. 6:125. doi: 10.3389/fnagi.2014.00125

Luque-Casado, A., Perakakis, P., Hillman, C. H., Kao, S. C., Llorens, F., Guerra, P., et al. (2015). Differences in sustained attention capacity as a function of aerobic fitness. Med. Sci. Sports Exerc. doi: 10.1249/mss.0000000000000857 [Epub ahead of print].

Madden, D. J., Costello, M. C., Dennis, N. A., Davis, S. W., Shepler, A. M., Spaniol, J., et al. (2010). Adult age differences in functional connectivity during executive control. Neuroimage 52, 643-657. doi: 10.1016/j.neuroimage.2010.04.249

Madden, D. J., Spaniol, J., Whiting, W. L., Bucur, B., Provenzale, J. M., Cabeza, R., et al. (2007). Adult age differences in the functional neuroanatomy of visual attention: a combined fMRI and DTI study. Neurobiol. Aging 28, 459-476. doi: 10.1016/j.neurobiolaging.2006.01.005

Markesbery, W. R. (2010). Neuropathologic alterations in mild cognitive impairment: a review. J. Alzheimers Dis. 19, 221-228. doi: 10.3233/jad-20101220

Meeuwissen, E. B., Takashima, A., Fernandez, G., and Jensen, O. (2011). Increase in posterior alpha activity during rehearsal predicts successful long-term memory formation of word sequences. Hum. Brain Mapp. 32, 2045-2053. doi: 10.1002/hbm. 21167

Minoshima, S., Giordani, B., Berent, S., Frey, K. A., Foster, N. L., and Kuhl, D. E. (1997). Metabolic reduction in the posterior cingulate cortex in very early Alzheimer's disease. Ann. Neurol. 42, 85-94. doi: 10.1002/ana.410420114

Moretti, D. V. (2015). Electroencephalography reveals lower regional blood perfusion and atrophy of the temporoparietal network associated with memory deficits and hippocampal volume reduction in mild cognitive impairment due to Alzheimer's disease. Neuropsychiatr. Dis. Treat. 11, 461-470. doi: $10.2147 /$ ndt.s78830

Nyberg, L., Lovden, M., Riklund, K., Lindenberger, U., and Backman, L. (2012). Memory aging and brain maintenance. Trends Cogn. Sci. 16, 292-305. doi: 10.1016/j.tics.2012.04.005

Oh, H., and Jagust, W. J. (2013). Frontotemporal network connectivity during memory encoding is increased with aging and disrupted by beta-amyloid. J. Neurosci. 33, 18425-18437. doi: 10.1523/jneurosci.2775-13.2013

Olson, R. L., Chang, Y. K., Brush, C. J., Kwok, A. N., Gordon, V. X., and Alderman, B. L. (2015). Neurophysiological and behavioral correlates of cognitive control during low and moderate intensity exercise. Neuroimage doi: 10.1016/j.neuroimage.2015.10.011 [Epub ahead of print].

Onoda, K., Ishihara, M., and Yamaguchi, S. (2012). Decreased functional connectivity by aging is associated with cognitive decline. J. Cogn. Neurosci. 24, 2186-2198. doi: 10.1162/jocn_a_00269

Park, D. C., and Reuter-Lorenz, P. (2009). The adaptive brain: aging and neurocognitive scaffolding. Annu. Rev. Psychol. 60, 173-196. doi: 10.1146/annurev.psych.59.103006.093656

Persson, J., Lustig, C., Nelson, J. K., and Reuter-Lorenz, P. A. (2007). Age differences in deactivation: a link to cognitive control? J. Cogn. Neurosci. 19, 1021-1032. doi: 10.1162/jocn.2007.19.6.1021

Petersen, R. C., Doody, R., Kurz, A., Mohs, R. C., Morris, J. C., Rabins, P. V., et al. (2001). Current concepts in mild cognitive impairment. Arch. Neurol. 58, 1985-1992. doi: 10.1001/archneur.58.12.1985

Petersen, R. C., and Morris, J. C. (2005). Mild cognitive impairment as a clinical entity and treatment target. Arch. Neurol. 62, 1160-1163; discussion 1167. doi: 10.1001/archneur.62.7.1160

Petersen, R. C., Roberts, R. O., Knopman, D. S., Boeve, B. F., Geda, Y. E., Ivnik, R. J., et al. (2009). Mild cognitive impairment: ten years later. Arch. Neurol. 66, 1447-1455. doi: 10.1001/archneurol.2009.266

Petzinger, G. M., Fisher, B. E., McEwen, S., Beeler, J. A., Walsh, J. P., and Jakowec, M. W. (2013). Exercise-enhanced neuroplasticity targeting motor and cognitive circuitry in Parkinson's disease. Lancet Neurol. 12, 716-726. doi: 10.1016/s14744422(13)70123-6

Pineda-Pardo, J. A., Bruna, R., Woolrich, M., Marcos, A., Nobre, A. C., Maestu, F., et al. (2014). Guiding functional connectivity estimation by structural connectivity in MEG: an application to discrimination of conditions of mild cognitive impairment. Neuroimage 101, 765-777. doi: 10.1016/j.neuroimage.2014.08.002

Porto, F. H., Coutinho, A. M., Pinto, A. L., Gualano, B., Duran, F. L., Prando, S., et al. (2015). Effects of aerobic training on cognition and brain glucose metabolism in subjects with mild cognitive impairment. J. Alzheimers Dis. 46, 747-760. doi: 10.3233/jad-150033

Reddy, P. H., Manczak, M., Mao, P., Calkins, M. J., Reddy, A. P., and Shirendeb, U. (2010). Amyloid-beta and mitochondria in aging and Alzheimer's disease: implications for synaptic damage and cognitive decline. J. Alzheimers. Dis. 20(Suppl. 2), S499-S512. doi: 10.3233/jad-2010-100504

Reddy, P. H., and McWeeney, S. (2006). Mapping cellular transcriptosomes in autopsied Alzheimer's disease subjects and relevant animal models. Neurobiol. Aging 27, 1060-1077. doi: 10.1016/j.neurobiolaging.2005. 04.014

Rushworth, M. F., Walton, M. E., Kennerley, S. W., and Bannerman, D. M. (2004). Action sets and decisions in the medial frontal cortex. Trends Cogn. Sci. 8, 410-417. doi: 10.1016/j.tics.2004.07.009

Sala-Llonch, R., Bartres-Faz, D., and Junque, C. (2015). Reorganization of brain networks in aging: a review of functional connectivity studies. Front. Psychol. 6:663. doi: 10.3389/fpsyg.2015.00663

Sanabria-Diaz, G., Martinez-Montes, E., and Melie-Garcia, L. (2013). Glucose metabolism during resting state reveals abnormal brain networks organization in the Alzheimer's disease and mild cognitive impairment. PLoS ONE 8:e68860. doi: 10.1371/journal.pone.0068860

Schaie, K. W. (1994). The course of adult intellectual development. Am. Psychol. 49, 304-313. doi: 10.1037/0003-066X.49.4.304

Schilbach, L., Eickhoff, S. B., Rotarska-Jagiela, A., Fink, G. R., and Vogeley, K. (2008). Minds at rest? Social cognition as the default mode of cognizing and its putative relationship to the "default system" of the brain. Conscious. Cogn. 17, 457-467. doi: 10.1016/j.concog.2008.03.013

Schultz, S. A., Boots, E. A., Almeida, R. P., Oh, J. M., Einerson, J., Korcarz, C. E., et al. (2015). Cardiorespiratory fitness attenuates the influence of amyloid on cognition. J. Int. Neuropsychol. Soc. 21, 841-850. doi: $10.1017 / \mathrm{s} 1355617715000843$

Seeley, W. W., Crawford, R. K., Zhou, J., Miller, B. L., and Greicius, M. D. (2009). Neurodegenerative diseases target large-scale human brain networks. Neuron 62, 42-52. doi: 10.1016/j.neuron.2009.03.024

Seo, E. H., Lee, D. Y., Lee, J. M., Park, J. S., Sohn, B. K., Lee, D. S., et al. (2013). Whole-brain functional networks in cognitively normal, mild cognitive impairment, and Alzheimer's disease. PLoS ONE 8:e53922. doi: 10.1371/journal.pone.0053922

Smallwood, N., Spriggs, M. J., Thompson, C. S., Wu, C. C., Hamm, J. P., Moreau, D., et al. (2015). Influence of physical activity on human sensory long-term potentiation. J. Int. Neuropsychol. Soc. 21, 831-840. doi: 10.1017/s1355617715001095

Smith, J. C., Nielson, K. A., Antuono, P., Lyons, J. A., Hanson, R. J., Butts, A. M., et al. (2013). Semantic memory functional MRI and cognitive function after exercise intervention in mild cognitive impairment. J. Alzheimers Dis. 37, 197-215. doi: 10.3233/jad-130467

Sporns, O., and Zwi, J. D. (2004). The small world of the cerebral cortex. Neuroinformatics 2, 145-162. doi: 10.1385/ni

Stam, C. J. (2004). Functional connectivity patterns of human magnetoencephalographic recordings: a 'small-world' network? Neurosci. Lett. 355, 25-28. doi: 10.1016/j.neulet.2003.10.063

Stam, C. J., and van Straaten, E. C. (2012). The organization of physiological brain networks. Clin. Neurophysiol. 123, 1067-1087. doi: 10.1016/j.clinph.2012.01.011

Suzuki, T., Shimada, H., Makizako, H., Doi, T., Yoshida, D., Ito, K., et al. (2013). A randomized controlled trial of multicomponent exercise in older adults with mild cognitive impairment. PLOS ONE 8:e61483. doi: 10.1371/journal.pone.0061483

Suzuki, T., Shimada, H., Makizako, H., Doi, T., Yoshida, D., Tsutsumimoto, K., et al. (2012). Effects of multicomponent exercise on cognitive function in older 
adults with amnestic mild cognitive impairment: a randomized controlled trial. BMC Neurol. 12:128. doi: 10.1186/1471-2377-12-128

Tambini, A., Ketz, N., and Davachi, L. (2010). Enhanced brain correlations during rest are related to memory for recent experiences. Neuron 65, 280-290. doi: 10.1016/j.neuron.2010.01.001

Taubert, M., Lohmann, G., Margulies, D. S., Villringer, A., and Ragert, P. (2011). Long-term effects of motor training on resting-state networks and underlying brain structure. Neuroimage 57, 1492-1498. doi: 10.1016/j.neuroimage.2011.05.078

Tomasi, D., and Volkow, N. D. (2012). Aging and functional brain networks. Mol. Psychiatry 17, 471-558. doi: 10.1038/mp.2011.81

Turner, G. R., and Spreng, R. N. (2012). Executive functions and neurocognitive aging: dissociable patterns of brain activity. Neurobiol. Aging 33, 826.e1826.e13. doi: 10.1016/j.neurobiolaging.2011.06.005

van der Hiele, K., Bollen, E. L., Vein, A. A., Reijntjes, R. H., Westendorp, R. G., van Buchem, M. A., et al. (2008). EEG markers of future cognitive performance in the elderly. J. Clin. Neurophysiol. 25, 83-89. doi: 10.1097/WNP.0b013e31816a5b25

van Harten, A. C., Visser, P. J., Pijnenburg, Y. A., Teunissen, C. E., Blankenstein, M. A., Scheltens, P., et al. (2013). Cerebrospinal fluid Abeta42 is the best predictor of clinical progression in patients with subjective complaints. Alzheimers Dement. 9, 481-487. doi: 10.1016/j.jalz.2012. 08.004

van Uffelen, J. G., Chinapaw, M. J., van Mechelen, W., and Hopman-Rock, M. (2008). Walking or vitamin B for cognition in older adults with mild cognitive impairment? A randomised controlled trial. Br. J. Sports Med. 42, 344-351. doi: 10.1136/bjsm.2007.044735

Vecchio, F., Miraglia, F., Marra, C., Quaranta, D., Vita, M. G., Bramanti, P., et al. (2014). Human brain networks in cognitive decline: a graph theoretical analysis of cortical connectivity from EEG data. J. Alzheimers Dis. 41, 113-127. doi: 10.3233/jad-132087

Villemagne, V. L., Burnham, S., Bourgeat, P., Brown, B., Ellis, K. A., Salvado, O., et al. (2013). Amyloid beta deposition, neurodegeneration, and cognitive decline in sporadic Alzheimer's disease: a prospective cohort study. Lancet Neurol. 12, 357-367. doi: 10.1016/s1474-4422(13)70044-9

Voss, M. W., Chaddock, L., Kim, J. S., Vanpatter, M., Pontifex, M. B., Raine, L. B., et al. (2011). Aerobic fitness is associated with greater efficiency of the network underlying cognitive control in preadolescent children. Neuroscience 199, 166-176. doi: 10.1016/j.neuroscience.2011.10.009

Voss, M. W., Erickson, K. I., Prakash, R. S., Chaddock, L., Malkowski, E., Alves, H., et al. (2010a). Functional connectivity: a source of variance in the association between cardiorespiratory fitness and cognition? Neuropsychologia 48, 13941406. doi: 10.1016/j.neuropsychologia.2010.01.005

Voss, M. W., Prakash, R. S., Erickson, K. I., Basak, C., Chaddock, L., Kim, J. S., et al. (2010b). Plasticity of brain networks in a randomized intervention trial of exercise training in older adults. Front. Aging Neurosci. 2:32. doi: 10.3389/fnagi.2010.00032

Watts, D. J., and Strogatz, S. H. (1998). Collective dynamics of 'small-world' networks. Nature 393, 440-442. doi: 10.1038/30918

Wei, G. X., Dong, H. M., Yang, Z., Luo, J., and Zuo, X. N. (2014). Tai Chi Chuan optimizes the functional organization of the intrinsic human brain architecture in older adults. Front. Aging Neurosci. 6:74. doi: 10.3389/fnagi.2014.00074

Wei, G. X., Xu, T., Fan, F. M., Dong, H. M., Jiang, L. L., Li, H. J., et al. (2013). Can Taichi reshape the brain? A brain morphometry study. PLOS ONE 8:e61038. doi: 10.1371/journal.pone.0061038

Yeo, B. T., Krienen, F. M., Sepulcre, J., Sabuncu, M. R., Lashkari, D., Hollinshead, M., et al. (2011). The organization of the human cerebral cortex estimated by intrinsic functional connectivity. J. Neurophysiol. 106, 1125-1165. doi: 10.1152/jn.00338.2011

Zhou, B., Liu, Y., Zhang, Z., An, N., Yao, H., Wang, P., et al. (2013). Impaired functional connectivity of the thalamus in Alzheimer's disease and mild cognitive impairment: a resting-state fMRI study. Curr. Alzheimer Res. 10, 754-766. doi: 10.2174/15672050113109990146

Zhou, B., Yao, H., Wang, P., Zhang, Z., Zhan, Y., Ma, J., et al. (2015). Aberrant functional connectivity architecture in Alzheimer's disease and mild cognitive impairment: a whole-brain, data-driven analysis. Biomed Res. Int. 2015, 495375. doi: 10.1155/2015/495375

Zlatar, Z. Z., Towler, S., McGregor, K. M., Dzierzewski, J. M., Bauer, A., Phan, S., et al. (2013). Functional language networks in sedentary and physically active older adults. J. Int. Neuropsychol. Soc. 19, 625-634. doi: $10.1017 / \mathrm{s} 1355617713000246$

Conflict of Interest Statement: The authors declare that the research was conducted in the absence of any commercial or financial relationships that could be construed as a potential conflict of interest.

Copyright (c) 2016 Huang, Fang, Li and Chen. This is an open-access article distributed under the terms of the Creative Commons Attribution License (CC BY). The use, distribution or reproduction in other forums is permitted, provided the original author(s) or licensor are credited and that the original publication in this journal is cited, in accordance with accepted academic practice. No use, distribution or reproduction is permitted which does not comply with these terms. 